“Transfer” XV1: 1-2 (2021), pp. 1-18. ISSN: 1886-554

\title{
LA TRADUCCIÓN LITERARIA EN ARGENTINA: ESTUDIO DE CASOS
}

Adriana Cristina Crolla (ORCID 0000-0002-8043-3126)

Universidad Nacional del Litoral (Santa Fe, Argentina)

El proceso que denominamos traducción implica mucho más que la versión o conversión de un sistema codificado en otro. El lenguaje es un sistema comunicativo cuya dinámica, arbitrariedad, eventualidad y espontaneidad hace que el acto comunicativo interlingüe y transcultural trascienda lo denotado, lo normado y lo verbalizado en los textos meta. Y en cuanto a la traducción literaria, esta disparidad y hasta la "imposibilidad" traductiva, se potencia.

Por ello es siempre interesante analizar la incidencia del contexto en las elecciones que hacen los traductores literarios, así como el modo en que la misma acción traductiva inaugura tradiciones de lecturas o propone nuevas a producciones ya aclimatadas en la cultura meta.

En otras sedes (Crolla 2006; 2009; 2013b), afirmamos que, si a lo largo del tiempo la traducción habilitó la importación de un universo cultural "otro" a una cultura ajena en operación fundacional, convirtiendo espacios vacíos en unidades funcionales para la receptora, nos parecía pertinente enunciarlo con una ecuación triádica: traducción/tradición/tra-dicción, trasladando al último término, el plural valor del «tra-dictum» que toda traducción inaugura. Porque traducir es Trans-decir una distancia, Trans-decir una tradición y Trans-decir una lengua. ${ }^{1}$

Ya nadie niega, y el paradigma vigente lo afirma, que la traducción es la forma más privilegiada de leer. Lectura de signo plural y

\footnotetext{
${ }^{1}$ «Tra-dicción»: en su polisemia sonora parece contener tanto la falta de lo que se
} 
“Transfer” XV1: 1-2 (2021), pp. 1-18. ISSN: 1886-554

compartida que al socializarse -por circulación espontánea o por operaciones de la comunidad productora- acompañado de corpus de ensayo y crítica especializada, da como resultado la emergencia de una nueva tradición gestada en el «tra-dictum» que cada comunidad cultural aporta. Un nuevo campo intelectual con incidencia tanto en la comunidad receptora (enriquecida con nuevas destrezas interpretativas y sensibilidades estéticas) que también impacta en lo que se traduce.

Se establece así un fenómeno de doble valencia porque la cultura traducida, leída por la nueva, se reposiciona y transforma. Por tanto afirmamos que traducir es trans-decir culturalmente (Crolla 2006). Y en el proceso, el arte de la traducción literaria es en esencia una declaración sobre el valor de la propia literatura puesta en contacto y no en subordinación a la otredad. En esos términos, la traducción se postula como una operación infinita de lectura comparada.

\section{Cesare Civita, Boris Spivacow y el Centro Editor de América Latina}

Si pensamos en la historia de la traducción en Argentina, es importante reconocer el valor agregado que aportó la industria editorial durante el período de esplendor que se inicia hacia 1930. Momento en que la faraónica empresa de escolarización había logrado ya "argentinizar" gran parte de los hijos de inmigrantes y que el universo de lectores se había expandido y diversificado. Por esas épocas la industria editorial se transforma en autosuficiente y empieza a tener la capacidad de atender demandas que van desde la más alta cultura hasta revistas instructivas y de carácter popular.

Esta época es signada además por la llegada de importantes editores españoles que escapaban de la guerra civil y de la Segunda Guerra Mundial y que encararon en sus filiales argentinas de Espasa Calpe, Labor, Aguilar, Juventud, Sopena, sumadas a las de capital argentino como Losada, Emecé y Sudamericana, una enorme empresa 
“Transfer” XV1: 1-2 (2021), pp. 1-18. ISSN: 1886-554

traductiva que exportó desde Buenos Aires y durante casi cuatro décadas a América hispánica y a otros continentes, una parte importante de la literatura considerada canónica. Esta producción encontró cabida en el primado de un registro que, sin ser totalmente neutro, se acercaba bastante a las sonoridades y léxicos de un español un tanto alejado del peninsular. Registro que sentó las bases de un perfil lingüístico accesible y de gran plasticidad en un vasto territorio del universo hispanófono.

Los intelectuales y los empresarios de la cultura de entonces se hallaban bajo la absoluta convicción de que había que expandir el campo de las literaturas foráneas y para eso había que traducirlas. Porque ellas mismas preconizaban un nuevo modo de escribir y se hacía imperioso saber cómo se lograba aquello. Por lo que son en particular los escritores los que se proponen traducir no un contenido, sino sobre todo los recursos formales que revolucionaban la literatura del siglo XX.

Si nos posicionamos en el período condicionado por los efectos de la Segunda Guerra, que impidió a Europa la producción y exportación de libros, en Argentina se consolida un fantástico desarrollo de la industria editorial, basado en la interdisciplinariedad y la apertura de mercados. Razones que explican como durante el decenio peronista (1945-55), conducido en su mayoría por opositores al gobierno, el mundo editorial se expandiera enormemente: si en 1943 existían 69 casa editoriales, en 1944 se llega a 156.

Un hombre que incidió fuertemente en esa renovación fue Cesare Civita, italiano que escapa de Italia por su condición de judío, para primero radicarse en EEUU y trabajar para Walt Disney y luego radicarse en Argentina en 1941.

En Italia, antes de escapar, Cesare Civita había trabajado en la editorial Mondadori, experiencia que traslada a la Argentina revolucionando la política editorial local. Con sus emprendimientos pudo dar trabajo a otros exiliados impedidos de ejercer sus profesiones y a 
“Transfer” XV1: 1-2 (2021), pp. 1-18. ISSN: 1886-554

amigos argentinos cesanteados durante el decenio peronista, reuniendo un conjunto de voces disidentes en una experiencia original. Civita creó en la Argentina el imperio editorial Abril a partir de revistas de divulgación, comunicación y de historietas, a las que añadió una de automovilismo que se hizo famosa: Corsa, y otras varias, no menos populares -Siete Días, Panorama, Claudia, Adán- abarcando distintos rubros, hasta cubrir cabalmente todas las expectativas de un lector curioso que desea estar informado.

Civita fue activo participante de la Nuova Dante, asociación de antifascistas opositores a la cultura italiana oficial. Es allí donde conoce a Gino Germani (fundador de la Sociología en Argentina), otro italiano judío migrado por las leyes raciales, y le ofrece el puesto de subgerente editorial. Nace así un esplendor colaborativo al que se sumarán otros escritores y profesores excluidos de los centros de producción de conocimiento por motivaciones políticas, entre ellos José Spivacow. En 1941, cuando ingresa como corrector en Abril, ya conocía por ser su profesor privado de español, a Cesare Civita, Alberto Levy y Paolo Terni, fundadores de dicha editorial.

El perfil de editor que Spivacow, siguiendo a Civita, impone en los emprendimientos a su cargo es determinante para comprender la acción de difusión que alcanzan las propuestas editoriales en el gran público. Al caer el gobierno de Perón en 1955, Spivacow entra a trabajar en la Facultad de Ciencias Exactas de la Universidad de Buenos Aires donde en 1958 asume el rol de gerente de la recientemente creada editorial universitaria EUDEBA. Pero cuando en 1966 la dictadura del General Onganía interviene la universidad, una parte importante de los trabajadores en la editorial renuncian. Spivacow es uno de ellos. Y decide continuar su política de dotar de libros al alcance de cualquier bolsillo a la mayor cantidad de lectores, a través de mecanismos de comercialización accesibles. El lema "el libro es un artículo de primera necesidad y debe ser para todos". Lo consigue en un emprendimiento, el Centro Editor de América Latina que, a pesar de 
“Transfer” XV1: 1-2 (2021), pp. 1-18. ISSN: 1886-554

no contar con el financiamiento estatal y apoyo institucional universitario y de los diversos avatares económicos y políticos llega a publicar casi 5000 títulos, agrupados en 77 colecciones desde su fundación en 1966 y hasta 1995.

Los libros se vendían en los quioscos y las colecciones más vendibles solventaban los fracasos. Lo más importante fue el desafío de incorporar amplitud de temáticas y producciones de variado origen, y en ello, la literatura traducida tuvo un papel de relevancia.

Se ha dicho que ni Eudeba ni el CEAL fueron proyectos editoriales rentables; sin embargo, puede decirse que Spivacow llevó adelante empresas eficientes con el único objetivo que en verdad le importaba: "libros para todos, más libros para más". Un rasgo de inventiva de enorme relevancia para las prácticas docentes es que los las colecciones en fascículos eran acompañadas de libritos con las obras. Mientras los fascículos terminaban conformando tomos de colección: Biblioteca Universal (10 tomos); Literatura Argentina (4) y Literatura Contemporánea (5), los libritos, de costo accesible por la calidad del papel, se comercializaban también en modo independiente.

Todo ello permitió la lectura de literaturas foráneas, organizadas por siglos, géneros o lenguas de origen. Poemas, relatos y obras de teatro traducidos de la literatura oriental y occidental, desde los orígenes hasta la contemporaneidad, se canalizaron en traducciones realizadas por el equipo editorial o especialistas.

Un modo innovador entonces fue la selección en antologías de textos representativos que permitió acceder a novedad y variedad en la brevedad. Los fascículos, contaron también con modos novedoso de diseño y eficaz didactismo de su formulación. En sus páginas, las voces de expertos en la crítica, muchas en traducciones, se complementaban con fragmentos ilustrativos de los textos literarios.

Como afirmamos, fruto de ese enorme trabajo traductivo es la colección Capítulo Universal/Biblioteca Básica Universal, primera colección del CEAL que publicó literatura en traducción de manera ma- 
“Transfer” XV1: 1-2 (2021), pp. 1-18. ISSN: 1886-554

siva, nació en 1968, dirigida por Luis Gregorich con la asesoría literaria de Jaime Rest. Este eminente profesor de Literatura Inglesa, que compartió cátedra con Borges en la UBA, fue responsable de la selección, notas y traducción, en más de 15 libritos, de textos de Poe, V. Woolf, poetas medievales y románticos ingleses, Henry James, etc.

Por escasez de presupuesto se recurrió también a la reedición de traducciones de procedencia española. Por ello se detectan en algunos las marcas del registro español de origen junto a un español más neutro, que sin caer en la variedad rioplatense que por aquella época empieza a introducirse en la elección de traductores jóvenes, hace más amena la lectura a un lector local.

Un folleto mecanografiado hacia 1967, "Instrucciones para los traductores", da cuenta de la clara conciencia normativa que movía a estos editores. Junto a indicaciones de orden práctico se avanzan reflexiones sobre los modos a elegir a la hora de traducir:

Cualidades de la traducción: sobre todo, fidelidad. Ni quitar ni agregar nada arbitrariamente, respetando la adjetivación del original. No confundir, sin embargo, traducción fiel con traducción literal. La fidelidad al pensamiento del autor no debe lograrse a expensas de la corrección y libertad de expresión que da el dominio del español, que se descuenta en el traductor. (s/f:2. Subrayado en el original) (Falcon 2017)

Es innegable que se tenía clara conciencia de estar inaugurando una "tradición" de lecturas y que se estaba enriqueciendo y ampliando el espectro de la literatura local. Y que cada uno de los integrantes del CEAL trabajó en modo corporativo y formativo asumiendo múltiples funciones: redacción y edición de los fascículos, selección de los materiales, comentarios y también traducción.

La editorial representó el ideal de una pedagogía donde la traducción ocupó un mismo nivel de relevancia con la literatura producida en español (abandonando la política de neto predominio de la 
“Transfer” XV1: 1-2 (2021), pp. 1-18. ISSN: 1886-554

norma españolizante que había signado las primeras décadas del siglo).

El esplendor editorial de esos años tuvo enorme incidencia en la configuración de la figura del intelectual y en las prácticas de lectura. Esta expansión se correspondió con un período histórico y cultural inédito en el país, que combinó la modernización del campo intelectual, académico y científico con la emergencia de un público nuevo, variado y entusiasta.

Un sistema constitucional inestable, el revanchismo político y los conflictos sociales, no impedirán el vitalismo editorialista hasta la vuelta del peronismo en los inicios de los 70. Momento en que una quema nativa de la Biblioteca de Alejandría destruyó millares de libros en la sede de Centro Editor de América Latina en Buenos Aires y provocó el desmantelamiento de la Biblioteca Vigil de Rosario.

\section{Cesare Pavese: traduciendo la tradición santafesina}

Cesare Pavese, a quien homenajeamos en los 70 años de su trágica desaparición, tenía la convicción de que tener una tradición es menos que nada y que sólo buscándola se la puede vivir. Buscar una tradición, en los tempranos años 30 en la Italia ya signada por el fascismo, lo obligó a traducir, a leer operativamente una tradición "otra" para hacerla transitar y transignificar. Y al hacer visible los mecanismos de esa transposición, logró sacudir los cimientos anquilosados de la propia. Luego de las traducciones y lecturas críticas de Pavese sobre la literatura norteamericana, la literatura y la cultura italiana no volverán a ser iguales, sino "fatalmente" norteamericanas, es decir, vigorosas y maduras. Por su parte, el american way of life, signado por la tra-dicción empezada por Pavese, signará también un cambio en la tradición de origen.

Del mismo modo, la recepción que la intelectualidad y la academia santafesina otorgó al ideario y obra pavesiana, aportó un cam- 
“Transfer” XV1: 1-2 (2021), pp. 1-18. ISSN: 1886-554

bio radical en el gusto y en el modo de entender la literatura y la realidad.

En el seno del Instituto de Cinematografía de la UNL creado en 1958, tuvo cabida una experiencia notable a partir de un proceso de importación teórica de modelos literarios y culturales que renovaron el espectro ideológico. Una intensa actividad de lectura, comentario, traducciones y fundamentalmente discusiones en torno a la figura del escritor, del poeta, la función social de la poesía, así como valores ideológicos ligados al compromiso del escritor, reconfiguraron estos referentes.

Fundamentales fueron dos docentes, por entonces noveles escritores: Hugo Gola y Juan José Saer. Leer la Italia del momento, y en especial a Pavese, implicó para estos ávidos lectores de la literatura extranjera, comprometerse con la discusión que se había entablado en el seno de la cultura de izquierda entre la interdicción ideológica y la creación, la valoración de la expresión literaria y artística. Gola estaba marcado por una controversia similar desde su juvenil militancia política de izquierda y encontraba en Pavese un espíritu afín, ya que este también había adherido tempranamente al partido comunista. Por otra parte la obra e ideario de Pavese brindaba marco sustentable para contrarrestar la cerrazón de la estética literaria local.

En esa puesta en escena es fundamental y fundacional la traducción encarada por Rodolfo Alonso junto a Gola de los ensayos de Pavese, publicados bajo el título de El oficio de poeta por Nueva Visión, en 1957. Gesto inaugural también de Alonso como traductor incesante de la obra de Pavese. ${ }^{2}$

\footnotetext{
${ }^{2}$ Traducciones de Pavese: El oficio de poeta, Nueva Visión, Buenos Aires, 1957; Trabajar cansa / Vendrá la muerte y tendrá tus ojos. Prólogo de Marcelo Ravoni. Ed. Lautaro, Buenos Aires, 1961; Feria de agosto, Siglo Veinte, Buenos Aires, 1968; Poesía italiana contemporánea, Universidad de Carabobo, Valencia, Venezuela, 1970; Cuentos, CEAL, Buenos Aires, 1971; El coloquio del río y otros cuentos, CEAL, Buenos Aires, 1982. Los Mares del Sud y otros poemas. CEAL, 1982; Diarios de vida y obra de Cesare Pavese y
} 
“Transfer” XV1: 1-2 (2021), pp. 1-18. ISSN: 1886-554

\section{Rodolfo Alonso recordaba hace unos años:}

A mediados de los años 50, mi compañero algo mayor en la revista "Poesía Buenos Aires", el santafesino Paco Urondo, comenzó a invitarme a su ciudad natal. Así conozco entre otros a Hugo Gola, a un casi niño Juan José Saer y, cruzando el río hasta Paraná, en ese entonces todavía por lanchones, al inolvidable Juan L. Ortiz.

Por ese entonces, en Buenos Aires, Edgar Bayley me propone inaugurar la colección que dirige en la Editorial Nueva Visión, con un libro de Cesare Pavese. De inmediato pensé en compartir la propuesta con Gola: enseguida nos habíamos descubierto coincidiendo en nuestra devoción (más que admiración) por Pavese, que se había suicidado poco antes, en agosto de 1950... Seleccionamos y tradujimos juntos (en un clima de mutua y lúcida alegría, de compartido y apasionado descubrimiento) un volumen de ensayos de Cesare Pavese, al que pusimos por título El oficio de poeta, ${ }^{3}$ tomado de uno de los dos ensayos agregados por él mismo a la edición definitiva de su primer libro de poesía Lavorare stanca. Esos ensayos fueron incluidos en nuestra selección, pero sobre todo muchos de los que Italo Calvino había reunido en su primera edición, póstuma, de La letteratura ame-ricana e altri saggi. Y quizá, también, uno que otro de Feria d'agosto. (Alonso, en Crolla 2015:172-176)

En una entrevista reciente amplió sus recuerdos:

Elio Vittorini. CEAL, Buenos Aires, 1983; El oficio de poeta. Universidad Iberoamericana, México, 1994; Antología esencial. Fijando Vértigos, Buenos Aires, 2007; Mostra della poesia. Gli italiani./Muestra de la poesía. Los venezolanos. Bid \& Co. Ed., Caracas, 2008; Trabajar cansa /Vendrá la muerte y tendrá tus ojos. Alción, Córdoba, Argentina, 2011; El oficio de poeta. Prólogo y apéndice de Rodolfo Alonso. Duino, Buenos Aires, 2018.

${ }^{3}$ El oficio de poeta, de Cesare Pavese. Selección y traducción de Rodolfo Alonso y Hugo Gola. Editorial Nueva Visión, Buenos Aires, 1957, 114 páginas, con varias reediciones sucesivas. Se publica con el mismo título en la Universidad Iberoamericana, México, 1994, 148 pág., y en 2918 con prólogo y apéndice de R. Alonso en Duino, Buenos Aires. 
“Transfer” XV1: 1-2 (2021), pp. 1-18. ISSN: 1886-554

Antes de El oficio... ya se habían traducido aquí 5 novelas de Pavese, en 1952, 1953, 1954, 1956. ${ }^{4}$ Pero El oficio... fue la primera obra de envergadura teórica y su influencia, que me consta, se mantiene hasta la fecha, generación tras generación. La más reciente es de 2018 y agotó su pequeña tirada... Habría que aclarar que Nueva Visión no era una empresa comercial sino una rama del amplio movimiento de vanguardia que nos incluía y que Paco quiso reflejar en su Primera reunión de arte contemporáneo $o^{5}$ para la UNL, con la cual me llevó a colaborar. (Alonso agosto 2020)

Con respecto a los problemas con los que se topa el traductor, que no son solo de tra-dicción en la absoluta soledad de su acto, sino resultado de decisiones editoriales y de mercado que condicionan la selección, edición y difusión, manifiesta:

Marcelo Ravoni se encargó espontáneamente, sin yo saberlo, de entusiasmar a la Editorial Lautaro para traducir Trabajar cansa/Vendrá la muerte y tendrá tus ojos (1961). Por eso es suyo el prólogo. Dediqué allí mi labor a la memoria de Cesare Pavese. Lo malo es que no me dejaron ver pruebas de los dos libros de poemas, hay muchas erratas y se tragaron un poema. Les hice poner fe de erratas, pero eso nunca es perfecto, total.

Además dediqué décadas a trabajar sobre esa traducción, que igual tuvo éxito y mi nueva versión es de 2011 (Alción), con mucho éxito, que me sorprendió.

Antes Boris Schwarz, de Siglo XX, me había pedido traducir los "racconti" completos de Einaudi. Eran cientos y cientos de páginas.

\footnotetext{
${ }^{4}$ La luna y las fogatas (1952) Buenos Aires, Losada. Colección 'Los Grandes Novelistas de Nuestra Época', dirigida por Guillermo de Torre. Traducción de Romualdo Brughetti; El Compañero (1953) Buenos Aires: Editorial Lautaro. Traducción de A. V. (Alfredo Varela según Alonso); Entre mujeres solas (1954) Buenos Aires: Ed. Goyanarte. Traducción de Herman Mario Cueva; El hermoso verano (1956) Buenos Aires: Ed. Goyanarte. Traducción de Herman Mario Cueva.

${ }^{5}$ Para profundizar sobre este encuentro ver Crolla 2013 y AA.VV. 1958.
} 
“Transfer” XV1: 1-2 (2021), pp. 1-18. ISSN: 1886-554

Todavía usaba máquina de escribir, y una sola copia en carbónico. De pronto Schwarz edita Feria de agosto solo con los cuentos, sin los ensayos (que están en El oficio de poeta). Y se ve que le había dado algunos al CEAL, que edita una antología. Eso, más algunos que yo había publicado en grandes revistas que dirigí, como "Claudia"', "Karina", o quizá en otras literarias, es lo que se salvó. Tuve un divorcio y entre mucho libro que no pude llevar quedó mi copia de Cuentos de CEAL. Es la única pérdida que tengo con C. Pavese. (Alonso agosto 2020)

Zunilda Manavella, creadora de la cátedra de Literatura Italiana en el primer Profesorado de Letras de la UNL en 1970 y entusiasta lectora y difusora de Pavese en el medio, me obsequió su ejemplar. Con ella compartimos durante seis años el dictado al ser reincorporada en 1986 al concluir una injusta cesantía decretada en 1975 por la Triple A. La portada lleva su firma y el año de compra, 1971, que se corresponde a la edición por la Colección Biblioteca Básica Universal. Rodolfo Alonso, su traductor, tuvo la gentileza de dedicármelo durante una visita para la Settimana della Lingua Italiana nel Mondo, que organizara en Santa Fe en 2008.

Cuentos de Cesare Pavese, contiene 12 relatos y no incluye prólogo que aporte datos sobre la selección ni la traducción. El registro es amablemente local sin caer en el coloquialismo y se privilegia el "tu" sobre el "vos" rioplatense.

Las elecciones lingüísticas y de registros, así como la musicalidad interna, me fueron recientemente aclaradas por Alonso:

Yo no me propongo usar uno u otro. Me dejo guiar por el oído y el ritmo y el sentido del lenguaje que uso, que soy. Mi base debe ser el porteño rioplatense, pero matizado por mi oído que percibe sutilezas y cadencias hasta de lenguajes que no conozco. Ni España es unitaria en eso. ¿Quién habla, y dónde, y cuántos, hoy en "castizo"? Tienen por lo menos cinco lenguas oficiales.

\footnotetext{
${ }^{6}$ La revista fundada por Cesare Civita mencionada más arriba.
} 
“Transfer” XV1: 1-2 (2021), pp. 1-18. ISSN: 1886-554

Yo mismo soy hijo de inmigrantes gallegos y de infancia bilingüe. Y creo que en Italia cada aldea tiene su lengua propia. Y no sólo allí. Mi oído las acepta e incorpora, a todas (no sólo de Italia). En mi infancia porteña había tres lenguas del pueblo: lunfardo, vesre, jeringoso. En fin, es cosa de no acabar. (Alonso agosto 2020)

Un dato en la portada enuncia la labor traductiva: "versión española de Rodolfo Alonso". En otra entrevista recuperada por Internet, Alonso lo reconoce como una marca de época:

Uno de los Diálogos con Leuco [fue publicado] en el último número de la revista [Poesía Buenos Aires], que está dedicado a las musas. Pero la traducción de los poemas es un poco posterior. Ahora recuerdo que (Raúl Gustavo) Aguirre nunca ponía "traducción" en la revista. Siempre ponía "versión". Versión parece hacer referencia a algo personal, diferente a la idea de traducción que había en esa época. En realidad siempre es "una" traducción, nunca es "la" traducción. Pero "versión" no volví a ver que se usara. (Alonso 2012)

La traza que dejara la traducción y lecturas de Alonso, Gola y Saer en la tradición "Pavese", impactó decididamente en las propuestas curriculares del primer profesorado de Letras en el seno de la UNL. ${ }^{7}$

Leer, traducir y enseñar a Pavese en las aulas de la Universidad Nacional del Litoral de Santa Fe equivalió a un terrible escozor del que no se salió indemne. El primado que su obra ocupó durante al menos dos décadas, no volvió a ser ocupado por ningún otro escritor. Y todavía hoy es fuertemente recordada la tradición de tradiciones que sus lecturas inauguraran.

\footnotetext{
${ }^{7}$ Analicé en profundidad estos recorridos en el libro referido a la configuración de los espacios de la italianística en la universidad argentina y en particular modo en la universidad santafesina (Crolla 2013).
} 
“Transfer” XV1: 1-2 (2021), pp. 1-18. ISSN: 1886-554

\section{Traduciendo a Laura Pariani}

Laura Pariani me conmocionó desde el primer libro suyo que leí: Quando Dio ballava il tango (Rizzoli, 2002). No sólo por las particulares vicisitudes familiares y personales que determinaron para ella un destino "argentino", sino por la mirada oltreoceánica con que presentaba las problemáticas de la inmigración italiana en nuestro país, proponiéndonos una Argentina en cierto modo desconocida desde una perspectiva es/trábica (Crolla 2014), doblemente extranjera y local.

No tuve dificultades para incluirlo en mis programas de enseñanza de literatura italiana en el Profesorado de Italiano, pero no pude hacer lo mismo en Literatura italiana del Profesorado de Letras pues se necesitaba la mediación de la traducción.

Para mi sorpresa, Quando Dio ballava il tango no fue traducido en Argentina sino en España, y por una traductora sevillana: Patrizia Orts, radicada en una localidad italiana cerca del Lago d' Orta donde vive la escritora. Orts me confesó que se había sentido interesada en traducirlo porque hablaba de migración y de vivir entre lenguas: "Observo a diario lo que sucede con el idioma de los que, como yo, no viven en su propia casa" (Orts 2006). Por ello recurrió a la editorial valenciana Pre-Textos y entusiasmó al editor Manuel Borrás, quien, si bien le dio carta libre, le indicó que no quería un libro "argentino". Debió entonces limitar los argentinismos a los diálogos o monólogos, procurando siempre "no caer en el exceso". Para subsanar su propia extranjería y la de sus lecturas, recurrió a informantes argentinos y destinó este registro solo para los personajes inmigrantes de segunda generación y aquellos de primera que hubieran emigrado hacía mucho tiempo. Como su traducción iba dirigida a lectores europeos, incorporó al final del libro un glosario de términos argentinos.

En la misma entrevista, Patricia Orts mencionó que a Borrás le entusiasmaba publicar esa traducción porque "la editorial Alfaguara 
“Transfer” XV1: 1-2 (2021), pp. 1-18. ISSN: 1886-554

lo había rechazado poco antes diciendo que contenía incluso errores históricos y ique era intraducible!”. Decisión que pude corroborar en un encuentro con el lector de Alfaguara en Argentina, durante un congreso de Literatura Comparada en Río de Janeiro en 2007, quien me reconoció haberlo desestimado porque en el libro se hablaba del proceso inmigratorio de un modo "diferente y crítico", alejado del gusto del público argentino y reduciendo el volumen de lectores.

Lo que el Lector de Alfaguara no había evaluado apropiadamente, y así se lo manifesté, es que una historia tan "argentinamente localizada", traducida con tonalidades peninsulares, aseguraría a la editorial un número importante pero fugaz, de lectores hispanófonos de otros contextos, pero para el Río de la Plata resultaba en la pérdida de la mirada problematizante que nos propone su lectura.

Gabriela Romairone, tradujo en 2004 en La Plata, el multipremiado L uovo de Gertrudina (Rizzoli 2003). Texto todavía inédito:

Esta traducción contó a posteriori con la lectura y sugerencias del escritor Roberto Raschella, a quien siempre le voy a estar agradecida. Él avaló el texto en diferentes editoriales, habló por teléfono, me facilitó contactos. Todo fue en vano. La recepción fue buena y, en algún caso, no escatimaron elogios y pedidos de disculpas. Dejaron también entrever esperanzas y esto alargó los tiempos de espera y postergó la entrega en otra editorial. Pero en definitiva las excusas fueron similares: no contaban con fondos, no dependía de ellos porque la editorial respondía a capitales extranjeros. La impresión que me quedó fue que el mercado editorial en Argentina es pequeño y fuertemente dependiente. Las decisiones se toman en España o en Colombia, algo raro cuando se quiere publicar en Argentina para un público local. (Romairone $08 / 08 / 2020$ )

Por estas razones decidí hace unos años encarar la traducción de otro libro: Il piatto dell 'angelo (Giunti, Ed. 2013) junto a un equipo intercátedras de la universidad santafesina y de la Universidad Autónoma 
“Transfer” XV1: 1-2 (2021), pp. 1-18. ISSN: 1886-554

de Entre Ríos. ${ }^{8}$ Proyecto que luego de muchos derroteros fue finalmente acogido por el Centro de Ediciones de la UNL y se encuentra en su etapa final de publicación.

Además de la novela antedicha, con la intención de proponer una mirada más abarcadora del modo como la escritora ha transpuesto a la ficción sus intereses sobre la problemática, se seleccionaron dos cuentos del texto traducido por Romairone que tienen como protagonistas a monjas que migraron a la Argentina en diferentes épocas y contextos políticos, pero compartiendo loables vocaciones de servicio y trágicos destinos. Dos cuentos autobiográficos enviados por Pariani, completan la selección.

El equipo acordó criterios de traducción: 1) la elección del voseo, en un español no demasiado neutro sin caer en el pintoresquismo del lunfardo o del cocoliche, en atención a la fuerte impronta local y dialógica del estilo de Pariani; 2) el mantenimiento del artículo delante del nombre natural ya que, aunque incorrecto en el español fue trasplantado por la inmigración a la Pampa Gringa; 3) especial atención al estilo trilingüe de su escritura (italiano, dialecto, español). 4) Uso de cursivas para destacar el español en el original y en las expresiones dialectales, con inclusión de traducción y notas a pie de página; 5) mantenimiento de la conjunción subordinante "que" en aféresis, habitual en la variedad coloquial del español con valor explicativo o causal, por encontrar correspondencia con la forma "ché" (aféresis de poiché, perché, sicché, cosicché) que la autora elige

\footnotetext{
8 Integran el equipo Alberto Anunziato, profesor de «Traducción Literaria» en la UADER, Valeria Ansó, profesora de literatura italiana en los Profesorados de Italiano y Letras de la UADER y del Profesorado y Licenciatura en Letras de la UN, y la Profesora en Letras y especialista en Italiano, María Luisa Ferraris, con quien compartimos el Portal de la Memoria Gringa y las comisiones directivas de AMPRA (Asociación de Mujeres Piemontesas de la Argentina) y del Centro Piemontés de Santa Fe.
} 
“Transfer” XV1: 1-2 (2021), pp. 1-18. ISSN: 1886-554

como elemento distintivo de su estilo; 6) respeto a la adecuación lexical y semántica en el juego pendular entre lo culto y lo coloquial.

Para la perfecta interpretación y traducción de enunciados en dialecto o léxico coloquial, recurrimos a la escritora.

Un factor de indudable peso en un sistema literario, aunque en muchos casos invisible, es el de los académicos y didactas de la literatura. En la enseñanza de las literaturas traducidas, los docentes, de hecho, incidimos con nuestras elecciones en los posicionamientos. Y traduciendo, ampliamos la oferta.

Con respecto a la literatura y estudios sobre la migración en Argentina, en particular la italiana, falta recorrer un vasto camino para fundar una tradición transformadora gracias a la traducción. Es nuestro deber entonces comprometer nuestras acciones con una Tra/duc-di-dic/ción de textos que conformen una biblioteca de textualidades es/trábicas, basada en miradas propias y originales, sobre los procesos migrantes que nos gestaron.

Traducir a Pariani es trazo y trazo de este desafío.

\section{Referencias bibliográficas}

AA.VV. (1958). Primera reunión de arte contemporáneo, 1957. Instituto Social de la Universidad Nacional del Litoral, Santa Fe: UNL.

ALONSO, Rodolfo. (2015). "Con Cesare Pavese en Santa Fe”. En: Crolla (ed.). Italia y Francia en Santa Fe. diversidades, legados y reconfiguraciones. Edic. UNL, pp.172-176.

---. (2012). “Operación Pavese”. Revista N Literatura, Diario Clarín, 06/03/12. Accesible on line (última consulta: 30 de septiembre de 2020):

$<<$ https://www.clarin.com/literatura/cesare-pavese-rodolfoalonso-trabajar-cansa_0_ByFWyp8nvXx.html >> 
“Transfer” XV1: 1-2 (2021), pp. 1-18. ISSN: 1886-554

CROLLA, Adriana. (2015). "Presencia de lo italiano en la cultura y la intelectualidad santafesina y sus articulaciones con la UNL". En: CROLLA, Adriana. (ed.). Italia y Francia en Santa Fe. Diversidades, legados y reconfiguraciones. Santa Fe: Ediciones UNL, pp. 80-92.

---. (2013a). Leer y enseñar la italianidad. Sesenta años y una historia en la Universidad Nacional del Litoral. Santa Fe: Ediciones UNL.

---. (2013b). "Traducción literaria en Argentina. Tradición, matrices culturales y tra-dicciones en perspectiva comparada", TRANSFER, VIII: 1-15.

---.(2009). “Cesare Pavese, traductor de América, 'leedor' de tradiciones”. En: COSTA PICAZO, R. \& CAPALBO, A. (eds.). En el país de los sueños posibles, AAEA y FHUC-UNL. BMPress, pp. 35-50.

---.(2006). "La traduzione attraverso la tradizione e la tra-dizione culturale: il caso letterario argentino", HETEROGLOSSIA (Università di Macerata), 1-32.

FALCÓN, Alejandrina. (2017). "Hacia una historia de las traducciones y los traductores del CEAL: el caso de la Biblioteca Básica Universal (1968/1978)", El taco en la brea, no 4 (05/05/2017). Accesible on line (última consulta: 04 de septiembre de 2020): $<<$ https://ri.conicet.gov.ar/handle/11336/46196>>.

\section{Resumen:}

El trabajo aborda casos de interés de traducciones "situadas" e inaugurales en Argentina. El grupo editorial Centro Editor de América Latina dirigido por Spicacow que hace llegar la literatura universal a todos los bolsillos, Pavese traducido por la generación de 1960 de intelectuales ligados a la Universidad Nacional del Litoral y un desafío corporativo actual en la traducción de de Laura Pariani. 
“Transfer” XV1: 1-2 (2021), pp. 1-18. ISSN: 1886-554

Palabras clave: Tra/ducción/dición literaria; Centro Editor de América Latina; Pavese; Pariani; Argentina.

\title{
LiterARY TrANSLATION IN ARgENTINA: SOME CASE STUDIES
}

\begin{abstract}
:
It is always interesting to analyse the incidence of a given context over those texts that are meant to be translated, and its impact on the making of new reading traditions while proposing new perspectives on literary productions already anchored in the target culture. This said, this paper foregrounds interesting cases of situated and foundational translations in Argentina such as that of the "Centro Editor de América Latina" coordinated by Spicacow, who grants everyone access to universal literature through pocket editions. In the same way, the translation of "Pavese" by the 1960 generation of intellecttuals from "Universidad Nacional del Litoral", and a current corporate challenge through the translation of "Laura Pariani". Following this path, we will study these cases and their impact in Argentina.
\end{abstract}

Keywords: Literary Translation; Centro Editor de América Latina, Pavese; Pariani; Argentina. 